\title{
CLASSIFYING MAPS IN FIBERINGS OF HOMOGENEOUS BOUNDED DOMAINS
}

\section{SOJI KANEYUKI}

ABSTRACT. A homogeneous bounded domain in $\mathrm{C}^{n}$ admits a structure of a fiber space whose base and fibers are homogeneous bounded domains. For such a fibering there exists a universal fiber space with the same fibers, which plays an analogous role as a universal bundle does in topology. A sufficient condition is given in order that the canonical map of the base of the fibering into the classifying domain is injective. Some applications of it are also given.

It is known by $\mathrm{P}$ jateckii-Šapiro [4] that a homogeneous bounded domain $D_{0}$ admits a structure of a holomorphic fiber space over a homogeneous bounded domain $D_{2}$ with another homogeneous bounded domain $D_{1}$ as a standard fiber in such a way that the group of fiber-preserving holomorphic automorphisms is transitive on $D_{0}$. Such a fibering is called a homogeneous fibering of $D_{0}$. The universal and the classifying domains for a given homogeneous fibering are constructed also in [4] (see also [2]). In this note we give a sufficient condition $(P)$ in terms of the Lie algebra of the automorphism group of $D_{0}$ in order that the classifying map of $D_{2}$ into the classifying domain is injective. The condition $(P)$ is satisfied for the case where $D_{0}$ is an irreducible bounded symmetric domain. We consider also under what conditions a given fibering $D_{0} \rightarrow D_{2}$ is itself the universal fibering. Throughout this note we will employ basic terminologies and techniques in [4] and [2].

1. We recall notations in [4] and [2]. Let $\left\{t_{0}, j, \omega\right\}$ be a normal $j$-algebra. Then $t_{0}$ has a structure of a graded Lie algebra $t_{0}=t^{0}+t^{1}+t^{1 / 2}$ such that $t^{0}=j t^{1}, j t^{1 / 2}=t^{1 / 2}$ and there exists an element $r \in t^{1}$ satisfying $[j a, r]=a$ for all $a \in t^{1}$. This decomposition is called the canonical decomposition of $t$. In the sequel we put $t^{1}=R, t^{1 / 2}=W$. Let. $R=$ $\Sigma_{1 \leq i \leq k \leq p} R^{i k}, j R=\Sigma_{1 \leq i \leq k \leq p} R^{i k}$ and $W=\Sigma_{1 \leq i \leq p} W^{i}$ be the decompositions into root spaces, where $R^{i i}$ is one dimensional. The element $r$ can be uniquely written as $r=\Sigma_{1 \leq i \leq p} r_{i}\left(r_{i} \in R^{i i}\right)$. Let $\left\{\psi_{1}, \ldots, \psi_{p}\right\}$ be the dual base of the base $\left\{j r_{1}, \ldots, j r_{p}\right\}$ of $\Sigma_{i} j R^{i i}$. Then the subspaces $R^{i k}$, $j R^{i k}, W^{i}$ are the root spaces corresponding to the roots $\left(\psi_{i}+\psi_{k}\right) / 2$,

Received by the editors August 19, 1974.

AMS (MOS) subject classifications (1970). Primary 32M10, 32M15; Secondary 55F05, 53C 30.

Key words and phrases. Homogeneous bounded domains, bounded symmetric domains, $j$-algebras. 
$\left(\psi_{i}-\psi_{k}\right) / 2$ and $\psi_{i} / 2$ respectively. Every root of $t$ is one of the above ones. Note that the root spaces are the common eigenspaces of the operators ad $j r_{k}(1 \leq k \leq p)$.

Let $t_{1}$ be a $j$-ideal of $t_{0}$. Putting $h(x, y)=\omega([j x, y])+i \omega([x, y])$ $\left(x, y \in t_{0}\right), h$ is a positive definite hermitian form, and the orthogonal complement $t_{2}$ of $t_{1}$ with respect to $h$ is a $j$-subalgebra; we have the decomposition

$$
t_{0}=t_{1}+t_{2}
$$

Let $\mathrm{t}_{i}=j R_{i}+R_{i}+W_{i}(i=1,2)$ be the canonical decomposition. It is known that $\left[j R_{1}+R_{1}, t_{2}\right]=0$ and $\left[t_{2}, w_{1}\right] \subset W_{1}$. Let $g$ be the universal $j$-algebra of $t_{1}$ in the sense of [2]. Then we have the decomposition

$$
g=t_{1}+z
$$

where $z$ is the classifying $j$-algebra of $t_{1}$ which is not only a semisimple $j$-subalgebra of $g$, but a subalgebra of $g\left(W_{1}\right)$. The adjoint representation $\lambda$ of $t_{2}$ on $W_{1}$ is a $j$-homomorphism of $t_{2}$ into 8 , which is called the class. ifying j-homomorphism. $\lambda$ is naturally extended to a j-homomorphism $\tilde{\lambda}$ of $t_{0}$ into $g$ in such a way that $\tilde{\lambda} \mid t_{1}$ is the identity.

Let $D_{0}$ be a homogeneous bounded domain, $D_{0} \stackrel{D_{1}}{\rightarrow} D_{2}$ a homogeneous fibering, and $t_{i}(i=0,1,2)$ be the Iwasawa $j$-algebra of $D_{i}$. Then $t_{i}$ is normal. It is known [4] that $t_{1}\left(\right.$ resp. $t_{2}$ ) can be chosen to be a $j$-ideal (resp. $j$-subalgebra) of $t_{0}$ and that the decomposition (1) holds. Let $D$ and $D(8)$ be the homogeneous bounded domains corresponding to $g$ and 8 , which are called the universal and the classifying domains of $D_{1}$, respectively. $D$ has a structure of a fiber space over $D(z)$ with fibers isomorphic to $D_{1}$. We have the following commutative diagram [2]

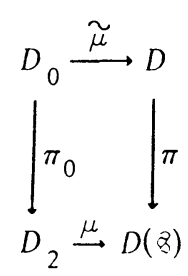

where $\pi_{0}$ and $\pi$ are the natural projections, and $\mu$ and $\tilde{\mu}$ are the holomorphic maps induced by $\lambda$ and $\tilde{\lambda}$ respectively. Note that $D(8)$ is a bounded symmetric domain.

2. Again consider the decompositions of $R, j R$ and $W$ into root spaces as in $\$ 1$. Then, from the arguments in [4, pp. 65, 70-71] and the uniqueness of canonical decompositions [1], it follows that 
(3)

$$
R_{1}=\sum_{1 \leq i \leq k \leq p_{1}} R^{i k}, \quad W_{1}=\sum_{1 \leq i \leq p_{1}} W^{i}+\sum_{1 \leq i \leq p_{1} ; p_{1}+1 \leq k \leq p}\left(R^{i k}+j R^{i k}\right),
$$

$$
R_{2}=\sum_{p_{1}+1 \leq i \leq k \leq p} R^{i k}, \quad W_{2}=\sum_{p_{1}+1 \leq i \leq p} W^{i}
$$

In the following, the indices $\alpha, \beta, \gamma, \ldots$ run through $1, \ldots, p_{1}$, and $\mu_{0}$, $\lambda, \mu, \nu, \ldots$ through $p_{1}+1, \ldots, p$.

Lemma $l_{。}$ Let $R^{\prime}=\Sigma_{a, \mu} R^{a \mu}$ and $W^{\prime}=\Sigma_{a} W^{a}$. Then, for $a, b \in R_{2}$, $u \in W_{2}$ we have

$$
\begin{array}{lll}
{\left[j a, w^{\prime}\right]=0,} & {\left[j a, R^{\prime}\right] \subset R^{\prime},} & {\left[j a, j R^{\prime}\right] \subset j R^{\prime},} \\
{\left[b, j R^{\prime}\right] \subset R^{\prime},} & {\left[u, w^{\prime}\right] \subset R^{\prime},} & {\left[u, j R^{\prime}\right] \subset W^{\prime} .}
\end{array}
$$

Proof. Let $a=\Sigma_{\mu, \nu} a_{\mu \nu}, a_{\mu \nu} \in R^{\mu \nu}$, and let $u \in W^{\alpha}$. If $\mu \neq \nu$, we have $\left[j a_{\mu \nu}, u\right]=0$, since $\left(\psi_{\mu}-\psi_{\nu}\right) / 2+\psi_{\alpha} / 2$ is never a root。 On the other hand $\left[j r_{\mu}, u\right]=\psi_{a}\left(j r_{\mu}\right) u / 2=0$, which implies $\left[j a_{\mu \mu}, u\right]=0$. Thus $\left[j a, W^{\prime}\right]=0$. The sum $\left(\psi_{\mu}-\psi_{\mu}\right) / 2+\left(\psi_{\alpha}+\psi_{\lambda}\right) / 2$ is a root if and only if $\lambda=\nu$, or $\mu=$ $\nu$. We have then $\left[j a_{\mu \nu}, R^{a \lambda}\right] \subset R^{a \mu}$ or $R^{a \lambda}$, according as $\lambda=\nu$ or $\mu=\nu$. This proves $\left[j a, R^{\prime}\right] \subset R^{\prime}$. Other relations are analogously proved.

We now introduce the following condition $(\mathrm{P})$;

for the subspace $\cdot R^{\prime}=\Sigma_{a, \mu} R^{a \mu}$ there exists an index

$\alpha\left(1 \leq \alpha \leq p_{1}\right)$ such that $R^{\alpha \mu} \neq(0)$ for each $\mu\left(p_{1}+1 \leq \mu \leq p\right)$.

Using its realization as a Siegel domain [5], [6], we can verify that (P) is satisfied for the Iwasawa $j$-algebra $t_{0}$ of an irreducible bounded symmetric domain and each nontrivial $j$-ideal $t_{1}$.

Lemma $\mathbf{A}$ [3]. For $x \in j R^{i k}(i<k)$ and $z \in U^{k}=W^{k}+\Sigma_{k<l}\left(j R^{k l}+R^{k l}\right)$, we have

$$
h([x, z],[x, z])=\lambda h(x, x) h(z, z),
$$

where $\lambda$ is a nonzero constant.

In Lemmas 2-4 we assume condition (P).

Lemma 2. If $\left[j a, j R^{\prime}\right]=0, a \in R_{2}$, then $a=0$.

Proof. Put $j a=\Sigma_{\mu \leq \nu} j a_{\mu \nu}, a_{\mu \nu} \in R^{\mu \nu}$. By (P) we can choose a nonzero element $j x_{\alpha \mu_{0}} \in j R^{a \mu_{0}}, \mu_{0}$ being an arbitrarily fixed index. From tbe hypothesis we have 


$$
\begin{aligned}
0=\left[j a, j x_{a \mu_{0}}\right] & =\sum_{\mu_{0} \leq \nu}\left[j a_{\mu_{0}} \nu, j x_{a \mu_{0}}\right] \\
& =\sum_{\mu_{0}<\nu}\left[j a_{\mu_{0} \nu}, j x_{a \mu_{0}}\right]+\left[j a_{\mu_{0} \mu_{0}}, j x_{a \mu_{0}}\right] .
\end{aligned}
$$

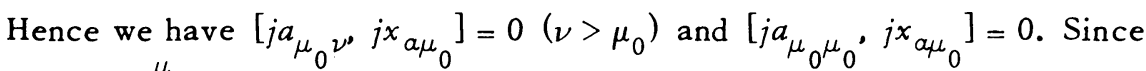
$j a_{\mu_{0} \nu} \in U^{\mu}$, we have, from Lemma $A, j a_{\mu_{0} \nu}=0$. On the other hand

$$
\begin{aligned}
{\left[j r_{\mu_{0}}, j x_{a \mu_{0}}\right] } & =j\left[j r_{\mu_{0}}, x_{a \mu_{0}}\right]+j\left[r_{\mu_{0}}, j x_{a \mu_{0}}\right] \\
& =j\left(1 / 2\left(\psi_{a}+\psi_{\mu_{0}}\right)\left(j r_{\mu_{0}}\right)\right) x_{a \mu_{0}}+j\left[r, j x_{a \mu_{0}}\right]=-1 / 2 j x_{a \mu_{0}},
\end{aligned}
$$

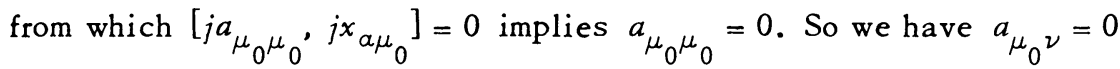
$\left(\nu \geq \mu_{0}\right) . \mu_{0}$ being arbitrary, we get $a=0$.

Using Lemma $A$, we have analogously:

Lemma 3. If $\left[u, j R^{\prime}\right]=0, u \in W_{2}$, then $u=0$.

Lemma 4. If $\left[b, j R^{\prime}\right]=0, b \in R_{2}$, then $b=0$.

Proof. Let $b=\Sigma_{\mu \leq \nu} b_{\mu \nu}, b_{\mu \nu} \in R^{\mu \nu}$. By (P) we can choose a nonzero element $j x_{\alpha \mu_{0}} \in R^{a \mu_{0}}, \mu_{0}$ being arbitrarily fixed. Then, from the hypothesis,

$$
\begin{aligned}
0=\left[b, j x_{a \mu_{0}}\right]= & \sum_{\mu_{0}<\nu}\left[b_{\mu_{0} \nu}, j x_{a \mu_{0}}\right]+\sum_{\mu<\mu_{0}}\left[b_{\mu \mu_{0}}, j x_{a \mu_{0}}\right] \\
& +\left[b_{\mu_{0} \mu_{0}} j x_{a \mu_{0}}\right] .
\end{aligned}
$$

So we get $\left[b_{\mu_{0} \nu}, j x_{a \mu_{0}}\right]=0\left(\nu>\mu_{0}\right)$ and $\left[b_{\mu_{0} \mu_{0}} j x_{a \mu_{0}}\right]=0$. Using Lemma $A$ and an argument similar to that in Lemma 2, we conclude $b_{\mu_{0} \nu}=0$ $\left(\nu \geq \mu_{0}\right) . \mu_{0}$ being arbitrary, $b=0$.

Proposition 1. Let $t_{0}$ be a normal j-algebra and $t_{1}$ a nontrivial $j$ ideal of $t_{0}$ and $t_{0}=t_{1}+t_{2}$ be the decomposition (1). Suppose condition (P) is satisfied for $t_{0}$ and $t_{1}$. Then the classifying $j$-homomorphism $\lambda$ of $\mathrm{t}_{2}$ into 8 is injective.

Proof. Take an element $x=j a+b+u \in t_{2}\left(a, b \in R_{2}, u \in W_{2}\right)$, and suppose $\left[x, W_{1}\right]=0$. Then, by (3) we have

$$
\left[j a, W^{\prime}\right]+\left[j a, R^{\prime}\right]+\left[j a, j R^{\prime}\right]+\left[b, j R^{\prime}\right]+\left[u, W^{\prime}\right]+\left[u, j R^{\prime}\right]=0 .
$$

Therefore, by Lemma 1 we have

$$
\left[j a, j R^{\prime}\right]=0, \quad\left[u, j R^{\prime}\right]=0, \quad\left[j a, R^{\prime}\right]+\left[b, j R^{\prime}\right]+\left[u, W^{\prime}\right]=0 .
$$

In view of Lemmas 2-4, we get $x=0$. 
Theorem 1. Let $D_{0} \stackrel{D_{1}}{\rightarrow} D_{2}$ be a homogeneous fibering of a homogeneous bounded domain $D_{0}$. If condition $(\mathrm{P})$ is satisfied for the Iwasawa $j$-algebra $t_{0}$ of $D_{0}$, then the classifying map $\mu$ of $D_{2}$ into $D(B)$ is injective, in particular, $\mu$ is a holomorphic imbedding.

Proof. We will first recall the definition of $\mu$ [2]. Let $T_{2}$ be the simply connected Lie group corresponding to $t_{2} . D(Z)$ is represented as the coset space $S / K$, where $S$ is a connected Lie group whose Lie algebra is $B$ and $K$ is the (compact) isotropy subgroup at a point in $D(B)$. The homomorphism $\lambda$ of $t_{2}$ into $\delta$ is extended to a homomorphism of $T_{2}$ into $S$, which is denoted again by $\lambda$. Since $T_{2}$ acts on $D_{2}$ simply transitively, $y \in D_{2}$ is uniquely written as $y=t \cdot o$, where $t \in T_{2}$ and $o$ is a fixed point of $D_{2}$. Then $\mu$ is defined by $\mu(y)=\lambda(t) K$. Take two points $y, y^{\prime} \in D_{2}$ and let $y=t \cdot o, y^{\prime}=t^{\prime} \cdot o\left(t, t^{\prime} \in T_{2}\right)$. Suppose $\mu(y)=\mu\left(y^{\prime}\right)$. Then $\lambda\left(t^{-1} t^{\prime}\right) \in K$. On the other hand $\lambda\left(T_{2}\right)$ is $\mathbf{R}$-triangular [2] and $K$ is compact, so $\lambda\left(t^{-1} t^{\prime}\right)$ is the identity. By Proposition $1, \lambda$ is injective, since $\lambda\left(T_{2}\right)$ is simply connected, and so $y=y^{\prime}$. It is obvious that $\mu$ is a holomorphic imbedding.

As was remarked before, condition $(\mathrm{P})$ holds when $D_{0}$ is irreducible symmetric. So we have

Corollary. Let $D_{0}$ be an irreducible bounded symmetric domain. Then, for any nontrivial homogeneous fibering of $D_{0}$, the classifying map $\mu$ is a holomorphic imbedding.

Theorem 2. Under the assumptions of Theorem $1, D_{0}$ is itself the universal domain of $D_{1}$ provided that $\operatorname{dim} D_{2}=\operatorname{dim} D(8)$.

Proof. $\mu\left(D_{2}\right)$ is the $\lambda\left(T_{2}\right)$-orbit of the origin of $D(8)=S / K$. From the fact that $\mu$ is an imbedding (Theorem 1) and $\operatorname{dim} D_{2}=\operatorname{dim} D(B)$, it follows that $\mu\left(D_{2}\right)$ is open in $D(z)$. Since $D(B)$ is connected homogeneous Kählerian, $\mu\left(D_{2}\right)$ must coincide with $D(8)$. So the $\mathbf{R}$-triangular subalgebra $\lambda\left(t_{2}\right)$ is a maximal R-triangular subalgebra of $\delta_{\text {. }}$. Therefore the subalgebra $t_{g}=t_{1}+\lambda\left(t_{2}\right)$ is seen to be an Iwasawa j-algebra of $D[2]$. So $\tilde{\lambda}$ is a $j$-isomorphism of $t_{0}$ onto $t_{g}$, which implies that $D_{0}$ is holomorphically isomorphic to $D$.

Remark. The following facts are verified by using Theorem 2 and [5]: A classical domain $D_{0}$ of type $I$ is universal if and only if the fiber $D_{1}$ is not isomorphic to a unit open ball. A classical domain $D_{0}$ of type II or III is always universal, while that of type IV is never universal if $\operatorname{dim} D_{0}$ $>3$. 


\section{REFERENCES}

1. S. Kaneyuki, On the automorphism groups of homogeneous bounded domains, J. Fac. Sci. Univ. Tokyo Sect. I 14 (1967), 89-130. MR 37 \#3056.

2. - Homogeneous bounded domains and Siegel domains, Lecture Notes in Math., vol. 241, Springer-Verlag, Berlin, 1971.

3. I. I. Pjateckii-Šapiro, The geometry and classification of bounded homogeneous regions, Uspehi Mat. Nauk 20 (1965), no. 2 (122), 3-51 = Russian Math. Surveys 20 (1965), no. 2, 1-48. MR 33 \# 4323.

4. - Automorphic functions and the geometry of classical domains, Fizmatgiz, Moscow, 1961; English transl., Math. and its Applications, vol. 8, Gordon and Breach, New York, 1969. MR 25 \# 231; 40 \# 5908.

5. - Geometry of classical domains and theory of automorphic functions, Fizmatgiz, Moscow, 1961; French transl., Dunod, Paris, 1966. MR 25 \#231; 33 \# 5949.

6. T. Tsuji, On infinitesimal automorphisms and homogeneous Siegel domains over circular cones, Proc. Japan Acad. 49 (1973), 390-393.

DEPARTMENT OF MATHEMATICS, NAGOY A UNIVERSITY, NAGOYA, JAPAN 